\title{
Analysis of bone cement distribution around fenestrated pedicle screws in low bone quality lumbosacral vertebrae
}

\section{AQ1}

Sergic AQ2 Gómez González, 1

Gerard Cabestany Bastida, 1

Maria Daniela Vlad, 1,2

José López López, 1

Pablo Buenestado Caballero, 3

Luis Alvarez-Galovich, 4

Maria Rodríguez-Arguisjuela, 4

Enrique Fernández Aguado, 1四

Email enrique.fernandez@upc.edu

1 Research Group of Interacting Surfaces in Bioengineering and Materials Science (InSup), Technical University of Catalonia (UPC), Avda. Diagonal 647, 08028 Barcelona, Spair AQ3

2 Faculty of Medical Bioengineering, "Grigore T. Popa" University of Medicine and Pharmacy Iasi, Str. Kogalniceanu 9-13, 700454 Iasi, Romania

3 Department of Mathematics, Technical University of Catalonia (UPC), Avda. Eduard Maristany 16, 08019 Barcelona, Spain

4 Spine Unit, Hospital Universitario Fundación Jimenez Díaz, Avda. Reyes Católicos 2, 28040 Madrid, Spain

Received: 7 May 2018 / Accepted: 13 August 2018

\section{Abstract}




\section{Purpose}

To study the exact distribution of bone cement around augmented fenestrated pedicle screws in both lumbar and sacral vertebrae of patients with low bone quality.

\section{Methods}

A total of 37 patients with instrumented lumbar fusion were investigated. 3D computed tomography virtual models of the injected cement and screws were obtained. The models were computed for their centroid (i.e. their average mass centre point), and their coordinates $(x, y, z)$ were projected on their respective screw-transversal and screw-longitudinal planes for further analysis.

\section{Results}

The results showed better bone cement homogeneous distribution around the screws in lumbar (L4 and L5) than in sacral (S1) vertebrae. In the lumbar region, the centroids were transversally projected near the transversal centre of symmetry of the screws. On the other hand, in the sacral region, the cement flowed preferentially outside the centre of symmetry of the screws, into the sacral ala.

\section{Conclusions}

The results confirm the different flow behaviours of bone cement in lumbar versus sacra vertebrae. The computer methodology followed in this study helps to understand the clinical monitoring observations and lays the foundations for better positioning of the screws and specific vertebrae-oriented screw designs.

\section{Keywords}

Spine screws

Cement augmentation

Pedicle screw augmentation

Computed tomography- $\mathrm{CT}$

Computer assisted design-CAD

\section{Introduction}


Spine fusion surgery procedures using transpedicular fixation have risen in the last decades. The increase in life expectancy of modern society, where elderly population form an ever-greater proportion, is directly related to that trend. Thus, the complications and outcome of this sort of surgery concerning elder people affected by severe degenerative spinal diseases and osteoporosis have become the topic of a number of investigations [1,2]. Additionally, complications during spine fusion procedures on lumbar and sacral vertebrae are more likely to appear on elderly patients than on any other segment of the population. And of special interest and relevance is the risk related to osteoporotic bone fixation $[3,4,5,6]$. For instance, a revision of the literature reveals a linear correlation between pull out strength of pedicle screws and bone mineral density $[7,8]$.

In order to reduce the overall impact of these complications, cement augmentation of pedicle screw fixation has become a used technique for providing spine stability. It has been reported that this procedure can increase screw pull out strength by a factor of up to 1.5 compared to surgical procedures that employ non-augmented screws $[9,10]$. Such has been its contribution to surgical success, that, currently, it has become a common implant used in spinal surgery, providing superior spine stability in elderly patients with osteoporosis and various spinal diseases $[11,12$, $13,14,15]$.

In any case, there still are a number of factors that need to be taken into consideration if optimum spine stabilization is to be achieved through this method. First, regulation of both homogeneity and viscosity of the cement plays a fundamental role in assuring trabecular bone interdigitation and preventing any extra osseous cement leakage. Second, achieving symmetrical cement flow distribution around the pedicle screw has been recognized to improve screw fixation in vertebral body $[10,16,17,18]$.

There have been several studies assessing the increase in resistance to pull out and toggle failure in lumbar vertebrae yielded by the use of cement to augment pedicle screw fixation [19]. Yet, very few studies have been able to acknowledge this effect in vertebra sacra S1. Furthermore, most of the studies published in the field of cement augmentation of pedicle screws are experimental and few clinical reports on the application of these techniques in clinical practice are available. Moreover, as far as we know, there are no studies that show the polymethylmethacrylate (PMMA) distribution around fenestrated screws in clinical cases. In this sense, it is important to highlight that the objective of our clinical practice is to ensure the stability of all spinal instrumentation with cemented screws. From this point of view, it is important to ensure the best possible anchoring of the fixing screws. Experience shows, and other studies support it, that better fixation is obtained 
when a uniform distribution of cement around the fixation screws is achieved as the transfer of charge towards the surrounding bone tissue is improved. However, this situation is not obtained in clinical practice in all cases since the cement flows according to the specific porous microarchitecture of said tissues. Therefore, the focus of this study is to put on the table the reality of clinical practice both to understand what actually happens and to find more accurate solutions.

By means of clinical experience, we have seen that symmetrical cement distribution around the screws cannot always be obtained and it seems especially critical when treating sacral vertebrae. And with the bibliography supporting the idea that an asymmetrical distribution may compromise mechanical stability of the inserted screws, an analytical methodology to reliably characterize cement distribution around fenestrated pedicle screws in lumbosacral vertebrae has become a matter of high relevance. In agreement to the above considerations, the present study of clinical cases reveals for the first time, from medical images and quantifiable information, the exact three-dimensional distribution followed by bone cement around the fixation screws both in the lumbar vertebra and in the sacral vertebra. The study allows obtaining accurate comparative information between the different vertebrae. The knowledge of the exact flow patterns of the cement around the fenestrated screws should help to define a better positioning of these screws.

\section{Materials and methods}

\section{Study design and surgical procedure}

A total of 37 consecutive patients (27 females and 10 males, mean age 76 years, range 55-89 years) with low bone mineral density and various spinal diseases that underwent spinal instrumentation with cement augmented pedicle screws (after having signed informed consent, according to the protocols for human procedures approved by the Ethics Committee of the Instituto de Investigación Sanitaria Fundación Jiménez Díaz, Madrid, Spain) were selected for this study.

As an overview of the spinal diseases shown by the patients, 1 patient suffered from compression fractures (fractured vertebrae was not included in the study), 11 patients suffered from lumbar spondylolisthesis, 17 patients suffered from kyphoscoliosis, 1 patient suffered from kyphosis at the adjacent level with pseudarthrosis at previously fused level, 4 patients suffered from degenerative or adjacent discopathy and 3 patients suffered from lumbar stenosis. They underwent a standard, open, posterior midline approach to the lumbar spine. Patients with foraminal or central canal stenosis requiring aggressive decompression underwent laminectomy or hemilaminectomy, associated or not with facetectomy, prior to fusion using cannulated cemented pedicle screw instrumentation. Moreover, all 
patients were examined through dual energy X-ray absorptiometry (DEXA). A mean T-score on the hip of $-2.269(-4.4$ to -1$)$ was obtained, indicating low bone density. However, the final decision of augmenting the fenestrated screws was done intraoperative in case a weak attachment of the screw was observed.

The lumbosacral spine levels evaluated in the present work were located as follows: 27 L4 vertebrae, 24 L5 vertebrae and 21 S1 vertebrae. Each vertebra was treated bilaterally using cannulated fenestrated pedicle screws (Omega21 ${ }^{\mathrm{TM}}$, Biomet ${ }^{\circledR}$ Inc., Indiana, USA), and with the same augmentation technique as the other vertebrae, using vertebroplasty cement (Biomet V, Biomet ${ }^{\circledR}$ Inc., Indiana, USA). The cannulated fenestrated pedicle screws (with a diameter of $6.35 \mathrm{~mm}$ ) had a central canal without a distal opening and two small and two large holes located at 12 and $25 \mathrm{~mm}$ length from the tip. In the case of lumbar vertebrae (i.e. L4 and L5), 50- or 55-mm-length pedicle screws were used, while in the case of S1 vertebra, 40- or 45-mm-length pedicle screws were chosen. The maximum quantity of hand pressure injected cement, under fluoroscopy control, per screw was limited to $3 \mathrm{ml}$ for all cases. This could be a limitation of the study, but clinical practice always works under certain boundary conditions. In any case, all the patients were operated by the same team, in the same OR, and with the same protocol, as previously described [1].

The pedicle screw placement and cementation were carried out under $\mathrm{C}$-arm fluoroscopy. This intraoperative monitoring technique helped to locate the optimal starting pedicle point for screws and to check the hole through the pedicle created by the pedicle finder. It also contributed in controlling the angulation and position against the anterior vertebral wall of the inserted pedicle screws as well as controlling the distribution of the cement and any possible leakage. The placement of all screws was performed after removing the cortical bone at the pedicle entry site. After having created the hole, a standard rounded pedicle finder was guided with the help of fluoroscopy, so that its tip did not go beyond one third of the vertebral body. Then, a palpation of the bony wall was performed in order to check if the pedicle wall and the cortex of the vertebral body were intact. After that, it was taped with a 5.5-mm tape.

Once all cannulated pedicle screws were inserted with concentric angulation and checked for correct positioning, the instrumentation was completed and the screws were dynamometrically tightened. The cement was mixed and apportioned among three $1-\mathrm{cm}^{3}$ sterile syringes $\left(3 \mathrm{~cm}^{3}\right)$. These syringes fit in the screw's head and allowed for fine control over the flow of cement. When the cement reached a tooth paste-like viscosity, it was slowly injected through the screw. This operation proceeded under the same monitoring procedure as standard vertebroplasty, that is, 
continuous fluoroscopy so as to keep control of the distribution of the cement and any possible leakage. In case of leakage, cement injection was stopped and resumed a short time later. Figure 1 shows some pictures of the clinical procedure and its final outcome.

\section{Fig. 1}

Some steps of the surgical procedure. a Pedicle screws placed in position prior to cementation. b, $\mathbf{c}$ Injection of bone cement under continuous fluoroscopy control. d, e Final surgery outcome before and after putting in place the $1-\mathrm{cm}^{3}$ syringes for bone cement injection 


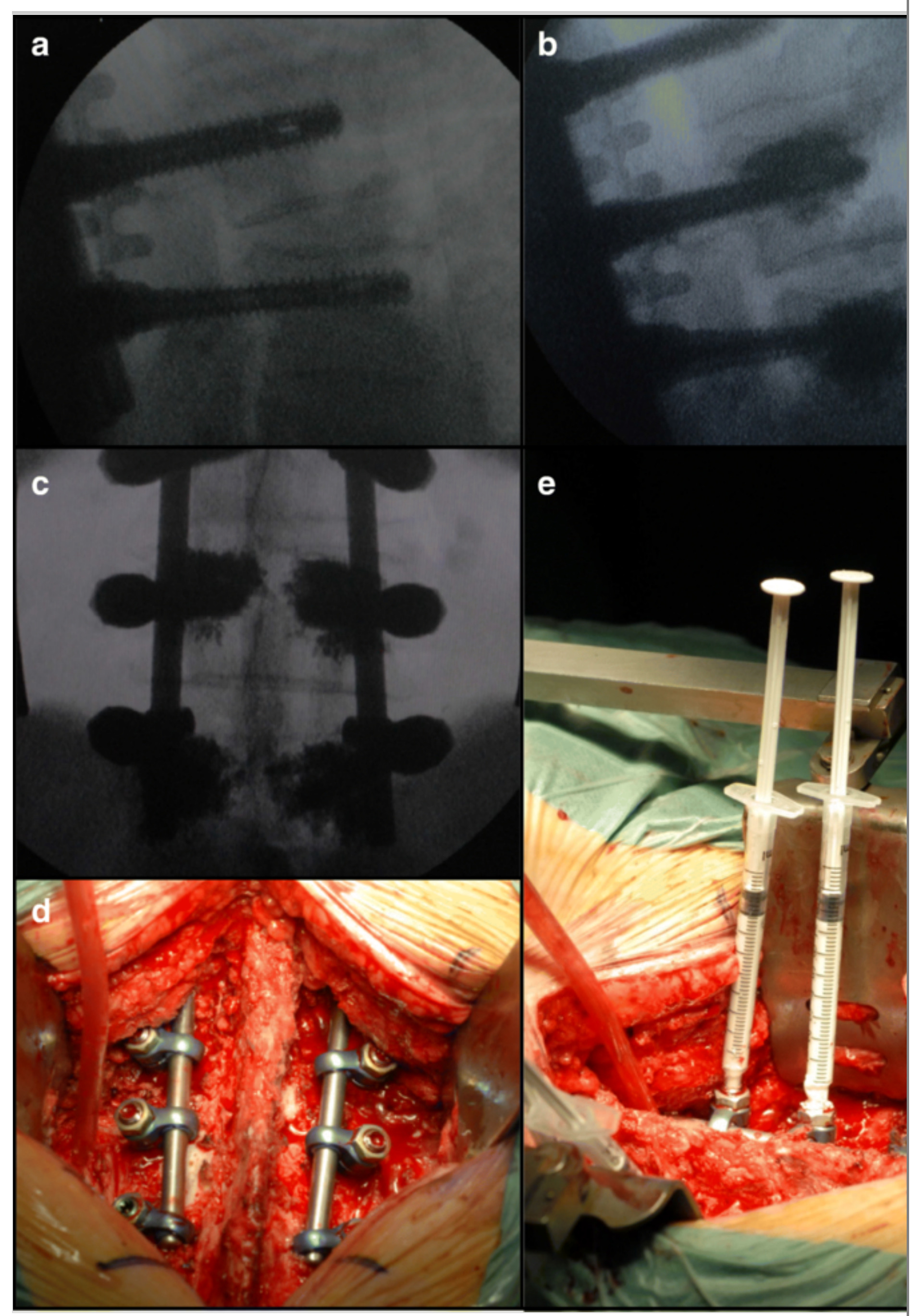




\section{Postoperative analysis: 3D model reconstruction and data acquisition}

A dual-energy multidetector CT scanner (Somatom Definition Flash, Siemens Medical Systems, Germany; $128 \times 2$ slices, layer thickness of $1.0 \mathrm{~mm}$, dual voltage of 80 and $140 \mathrm{kVp}$, current of 203 and $80 \mathrm{mAs}$, CareDose 4D and acquisition $32 \times$ $0.6 \mathrm{~mm}$ ) was used to monitor the patients once 6 months had passed after the operation. The CT files, in DICOM format, were imported to Invesalius 3.0 open source software ( http://www.cti.gov.br/invesalius/ ) for the three-dimensional (3D) reconstruction of the computed tomography images (see Fig. 2a-c). Before the 3D virtual reconstruction, images were properly treated with automated software tools for noise reduction, smoothing and segmentation. The 3D models showing the isolated screws and surrounding cement (see Fig. 2c) were then exported directly to Rhinoceros 3D(C) software (Robert McNeell \& Associates, v.4.0 SR9) as STL (Stereo Lithography) files (see Fig. 2d) in order to study bone cement distribution around each individual screw (see Fig. 2e). From that point, the Rotate software command was used to conveniently orient each screw (see Fig. 2f) so as to obtain the maximum longitudinal (see. Fig. 2g) and transversal (see Fig. 2h) 2D cement profile projections. Then, the MeshOutline software command was used to create a polyline outline profile of the polygon mesh objects for the projected surfaces (see also Fig. 2f). Then, the VolumeCentroid software command was used to report the coordinates $(x, y, z)$ of the centroid of the injected cement, that is, the average mass centre point of the bone cement distributed around the cannulated and fenestrated pedicle screws. Finally, in order to give a clear visual representation of the cement distribution and to ease its study and comprehension, the volumetric centroid (C) coordinates $(x, y, z)$ were assessed differentiating the ones corresponding to the longitudinal projection view $(x, y)$ (see Fig. $2 \mathrm{~g}$ ) and to the transversal projection view $(x, z)$ (see Fig. 2h). All this information was further CAD treated for optimum visual representation. In the end, an analysis of variance (ANOVA) using the obtained coordinates was performed to see if statistically significant differences between the behaviour shown by cement injected in different vertebrae were present.

\section{Fig. 2}

Experimental process followed to obtain the maximum sagittal and axial bone cement flow profiles around the spine screws. a, b 3D reconstruction of bone cement surrounding the screws in L4, L5 and S1 vertebrae (done with free open software InVesalius 3.0). c 3D isolated model for the screws and the surrounding cement. d 3D reconstructed model exported to Rhinoceros $(\mathrm{C}$ 3D in STL format. e Isolated 3D models for each screw. f CAD model and method used to obtain the maximum longitudinal and transversal 2D flow cement profiles. $\mathrm{g}$ CAD model showing the 
longitudinal view of the bone cement distribution. h CAD model showing the transversal view of the bone cement distribution

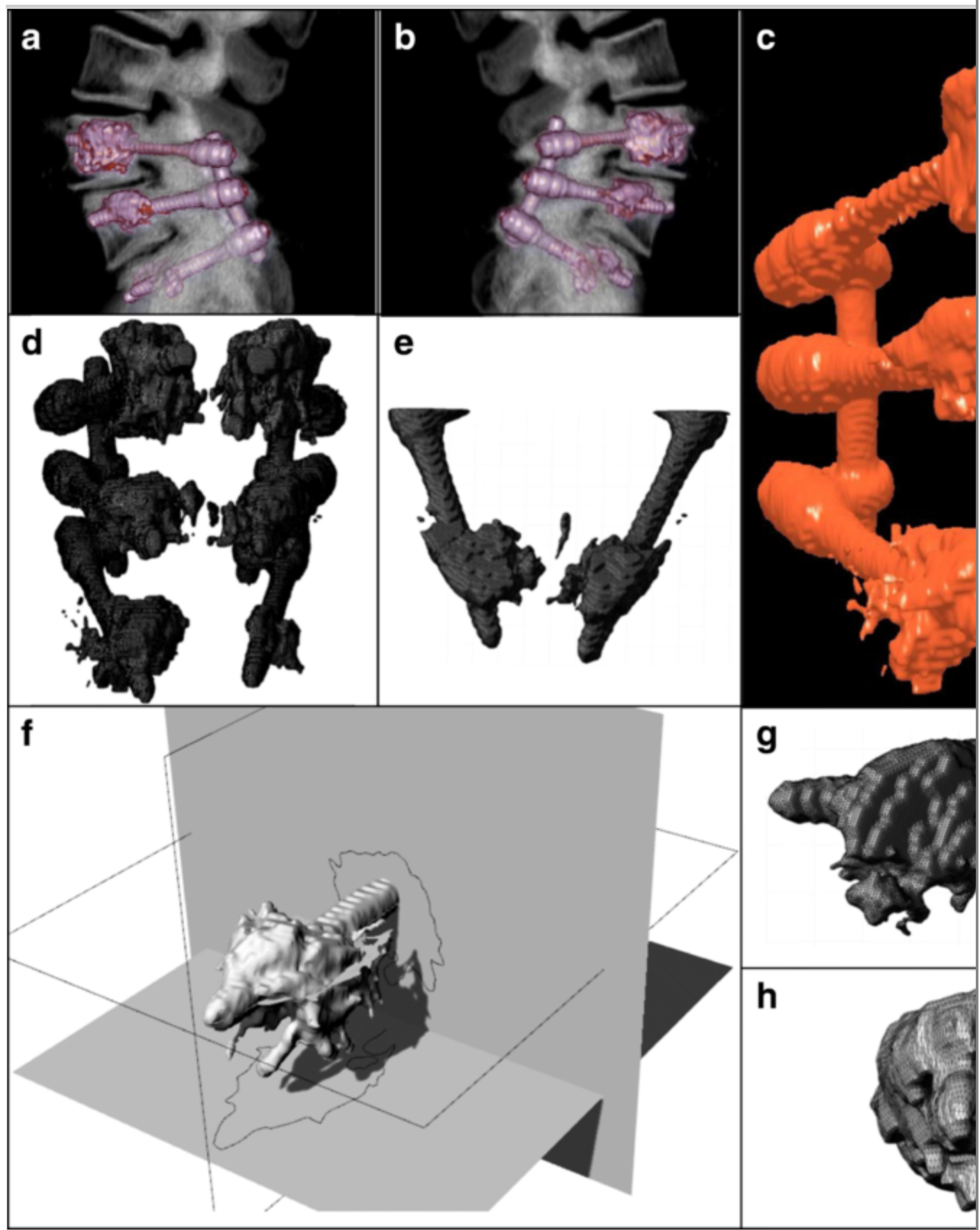

Results 
As explained in previous section, Fig. $2 \mathrm{f}$, which is the starting point for further data processing, shows a reconstructed CAD model of the pedicle screw with the injected cement, together with the cranium-caudal longitudinal and the anteroposterior transversal 2D-projected views of the maximum cement profiles. In this sense, Fig. 3 shows a set of these profiles (P) obtained for the pedicle screws inserted into the L4, L5 and S1 vertebrae of the same patient. It can be noticed that the main differences between pedicle screws inserted in separate vertebrae have strongly emerged when examining the transversal plots (see Fig. 3c, d). On the contrary, the longitudinal views (see Fig. $3 \mathrm{a}, \mathrm{b}$ ) have not shown such variation.

\section{Fig. 3}

Representation in polar coordinates of cement profiles $(\mathrm{P})$ and projected centroids (C) measured around spine screws inserted into the L4, L5 and S1 vertebrae of the same patient. a Cranium-caudal (CC) longitudinal view of the right screws. b CC longitudinal view of the left screws. c Antero-posterior (AP) transversal view of the right screws. d AP transversal view of the left screws
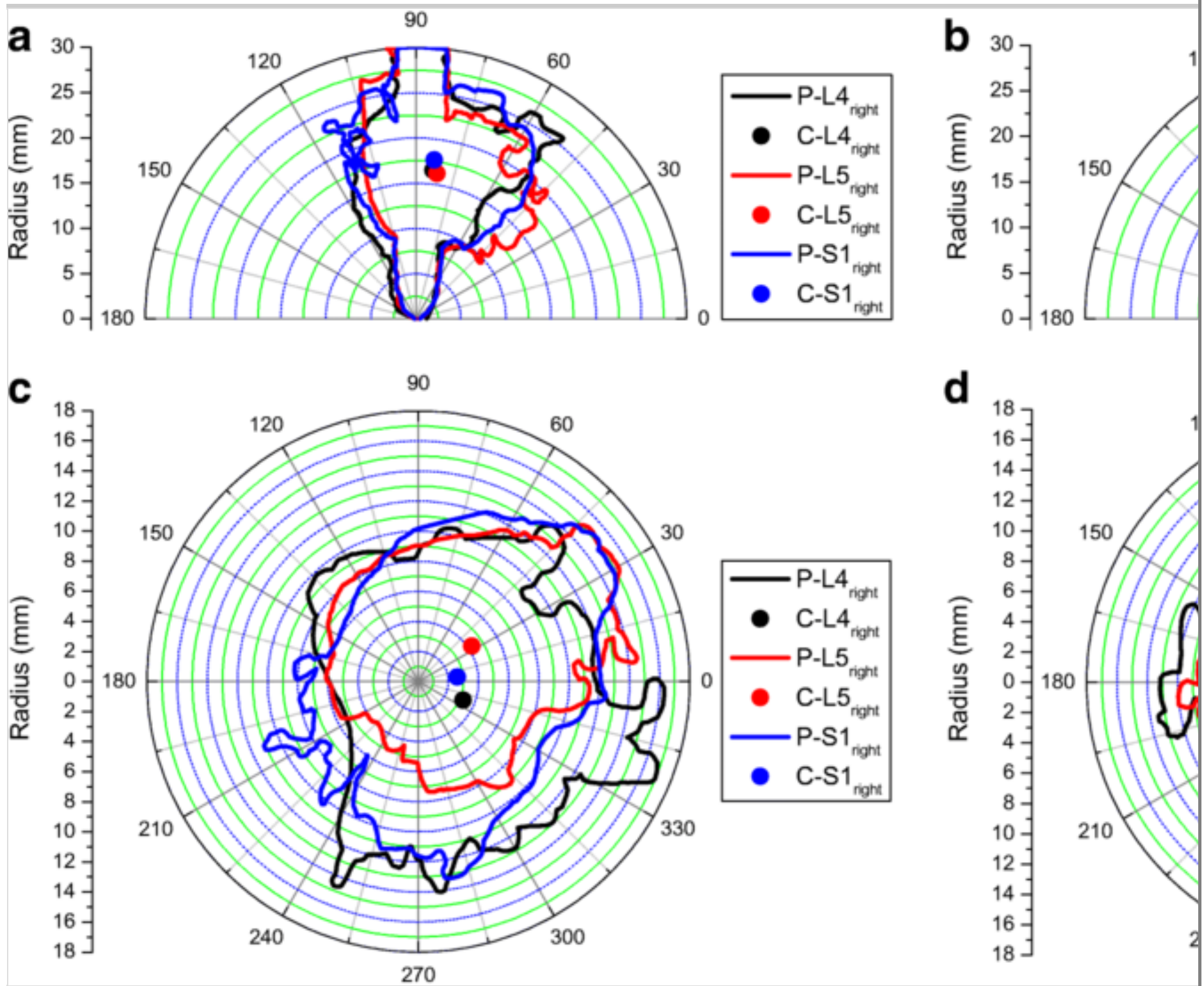
To better account for these differences, the 3D Cartesian coordinates of the centroids, projected on the screw symmetry longitudinal and transversal planes, have been represented jointly with the maximum projected profiles, in plane polar coordinates. These centre points allow for better visual differentiation between cement injected in different screws. For example, for a longitudinal view, Fig. 3a, b, while showing no significant differences between the L4, L5 and S1 vertebrae, i.e. the projected centroids nearly coincide, they do show an average tendency of the centroids to deviate towards the inner central part of the vertebrae for both the right (Fig. 3a) and the left (Fig. 3b) vertebrae implanted screws. This tendency is also captured by the transversal view (see Fig. 3c, d), which accounts for better differentiation between vertebrae. For that particular patient, the cement in the right screw deviates towards the centre-down and the centre-top parts of the L4 and L5 vertebrae, respectively. Meanwhile, cement injected in the right screw of S1 vertebra shows a more symmetric distribution. On the other hand, for the left screws, the cement injected in the S1 vertebra clearly deviates towards its downright wall, displaying a high asymmetrical distribution compared to a more symmetrical cement distribution shown by L4 and L5 vertebrae.

Figures 4 and 5 are graphic depictions of all the longitudinal (Fig. 4) and transversal (Fig. 5) projected centroid coordinates obtained for the whole set of analysed screws (a total of 27 for both L4 right and left; 24 for L5 right and 23 for L5 left; 21 for S1 right and 19 for S1 left) and intend to clarify the abovementioned particular observations. These figures allow for a proper study of cement distribution differentiating between both the right and the left screw insertion positions and the different vertebrae (L4, L5 and S1). For example, Fig. $4 \mathrm{a}-\mathrm{c}$ shows that the average projected centroid points of both the right and the left screws in L4, L5 and S1 vertebrae are aligned with the longitudinal symmetry screw axis and are allocated in between the positions of the two screw holes designed for cement flow (12 and $25 \mathrm{~mm}$ length from the screw tip). In polar coordinates, as those represented in Fig. 4, the average centroid points are aligned between radius $15-20 \mathrm{~mm}$ and angle $0^{\circ}$.

\section{Fig. 4}

Representation in polar coordinates of all the centroid points on the corresponding longitudinal cement projection profiles surrounding the spine screws inserted, right and left, into the L4 (a), L5 (b) and S1 (c) vertebrae of the population under study. Centroid average values for the right and the left series are also indicated $\mathbf{b}$ in polar coordinates of all the centroid points on the corresponding transversal cement projection profiles surrounding the spine screws inserted, right and left, into the L4 (a), L5 (b) and S1 (c) vertebrae of the population under study. Centroid average values for the right, the left and both together series are also indicated 

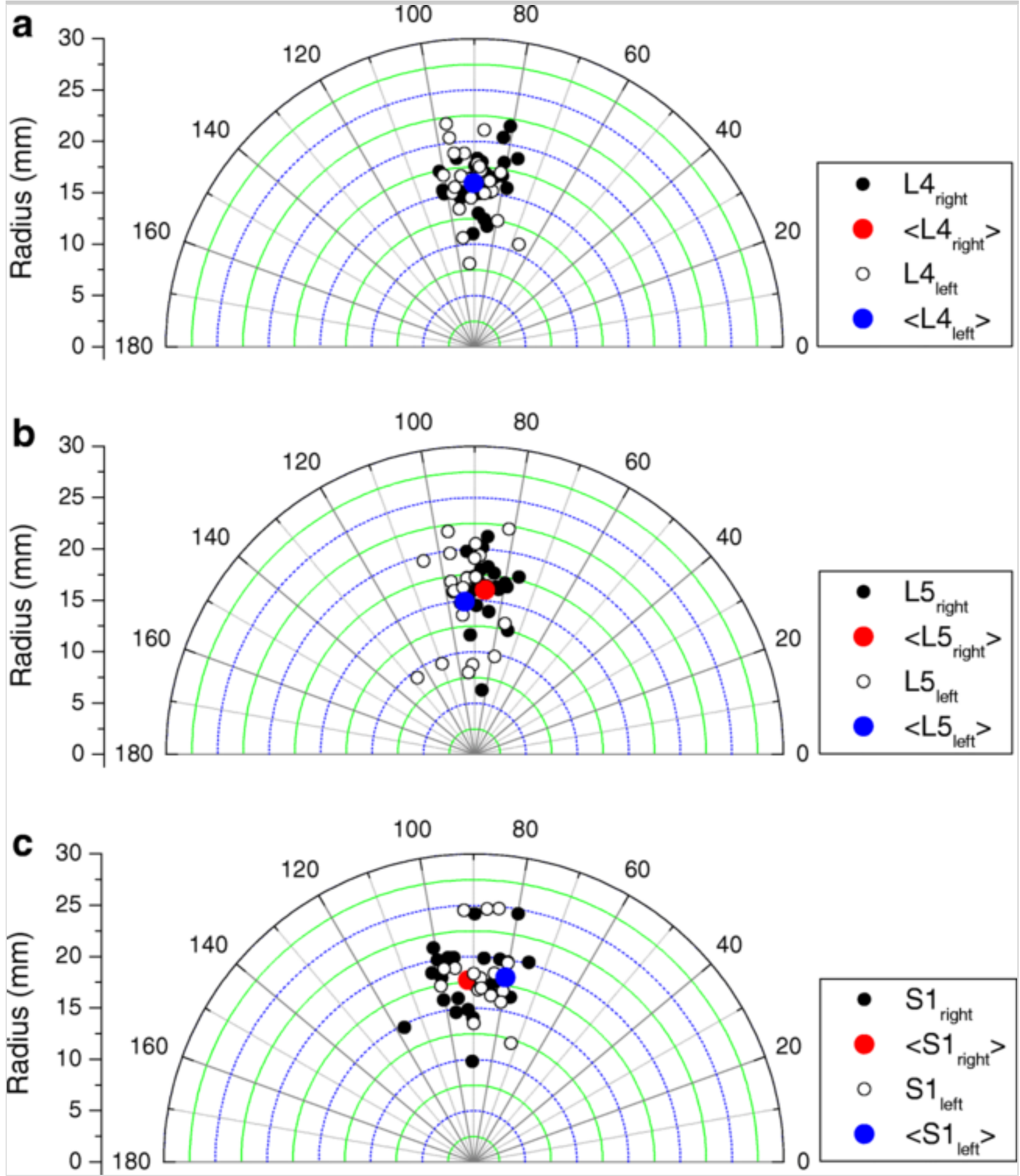

\section{Fig. 5}

Representation in polar coordinates of all the centroid points on the corresponding transversal cement projection profiles surrounding the spine screws inserted, right and left, into the L4 (a), L5 (b) and S1 (c) vertebrae of the population under study. Centroid average values for the right, the left and both together series are also indicated 

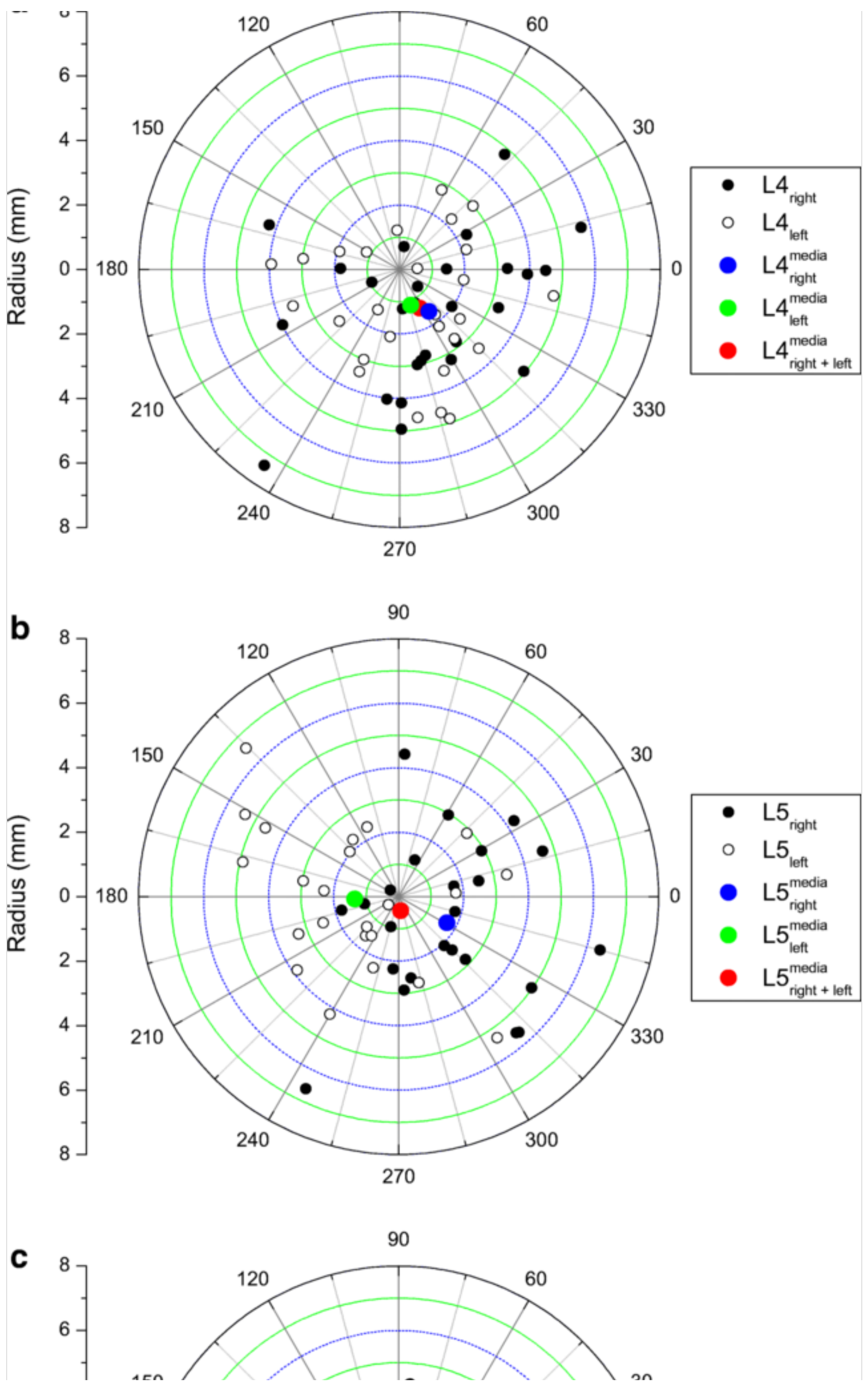
deviated the injected cement to the corresponding downside walls of the vertebra (note the position of the corresponding projected centroid points). The average Cartesian coordinates and the standard deviations of the centroids projected on the transversally projected profiles are the following: $(-0.369( \pm 3.26), 1.097( \pm$ $2.368))-(-1.897( \pm 2.675),-2.84( \pm 2.075)) \mathrm{mm}$. The one-factor ANOVA showed no statistically significant difference between the behaviour of injected cement in different screws $(p>0.05)$.

Lastly, the results obtained for the S1 vertebra are a clear indicator that cement injected into sacral S1 vertebra behaves differently from lumbar L4 and L5 vertebrae. While in lumbar vertebrae, the cement distributes mainly symmetrically around the screws; in the sacral vertebra, that is not the case and symmetrical and homogeneous cement distributions are not assured.

These observations were further confirmed by another statistical analysis of the data. In this case, the average values of the polar radius were assessed. The mean values and standard deviation of the radius of the joined populations were as follows: (a) for the L4 vertebra, $2.95( \pm 1.425) \mathrm{mm}$; (b) for the L5 vertebra, 3.025 $( \pm 1.636) \mathrm{mm}$; and (c) for the S1 vertebra, $4.49( \pm 2.191) \mathrm{mm}$. At the light of these results, the one-factor ANOVA clearly indicated that the S1 vertebra showed a statistically significant different $(p<0.05)$ behaviour as compared to the L4 and the L5 series. Additionally, the L4 and the L5 series did not show any statistical significance between them $(p>0.05)$.

\section{Discussion}

Succeeding in achieving a reliable fixation of the pedicle screw in vertebral body has been, and will be, the key element in assuring a successful outcome of cemented screws. In this sense, PMMA cement augmentation has been proved to enhance pedicle screw fixation in osteoporotic lumbar vertebral body $[13,19,20$, $21,22]$. The use of fenestrated screws allows applying a PMMA augmentation technique when a low bone quality is found. In this sense, the use of lumbar DEXA is not predictive in most cases of lumbar spine and especially in vertebra S1, since this value can be modified by arthritic changes, among others [23]. Several studies have evaluated the improvement in pedicle screw fixation in osteoporotic bone caused by the cement augmentation technique [12, 13, 14, 17]. However, very few of them have carried out a comparison between lumbar and sacral vertebrae [24, 25]. Much effort is put in this sort of surgery so as to achieve a homogeneous distribution, thus improving the probability of a successful fixation of the pedicle screw into the vertebra. And this study has allowed us to notice the existence of differences in behaviour between PMMA cement distribution in lumbar and sacral vertebra. 
In this study, it was seen that PMMA-injected cement distributed more uniformly around the inserted screws in lumbar (L4 and L5) vertebrae than in the case of sacral (S1) vertebra. As seen in Figs. 4 and 5, projected centroids, corresponding to both the longitudinal and the transversal cement profiles, were on average located in the lumbar region, close to the central geometric point of the screws. This should contribute, according to the literature, in favouring a better fixation by allowing the screw to have the same amount of cement in every direction around it, thus, avoiding any move or shift from its original position due to loosening of the screw. This performance behaves in agreement with the expected clinical outcome, where cement should fill the centre of the vertebra, avoiding approaching the walls.

On the contrary, projected centroids of the profiles of injected cement into the sacral vertebra (S1) showed a scattered distribution, displaying a preferential flow of the cement to the sacral ala. This leads to the possibility that injected cement found a preferential path towards the lateral walls instead of staying in the surroundings of the screw. The reasons for this are not yet clear but could be because of the different trabecular distributions of the sacral vertebrae (that lacks lateral wall) and the weaker ala that might allow PMMA to flow with less resistance [26] or because of the positioning of the screw. That last cause, i.e. the proximity of the screw to the lateral wall of the vertebra, could be affecting the eccentric distribution of cement in the sacral vertebrae in a more visible way than in lumbar vertebrae (see Fig. 5).

At the light of our results, it appears reasonable to state that the new CAD approach taken has succeeded in effectively providing reliable data that matches clinical observations. It confirms the statement made by the observation of clinical cases, regarding the asymmetrical distribution of cement injected in sacra vertebra (S1) compared to lumbar vertebrae. What is more, a highly detailed characterization of the cement distribution has now been achieved. And we hope that this will help build a better understanding regarding the behaviour shown by the injected cement into different vertebrae and contribute in improving augmentation techniques and devices that are currently in use. In this sense, the computer methodology followed in this study lays the foundations for better and specific vertebrae-oriented screw designs.

It should be highlighted that, despite biomechanical and finite element studies have a great clinical value, the aim of this study was to know the exact distribution of PMMA around the screws, especially in the sacrum, based on clinical cases. From this point of view, the flow pattern found for the cement clearly affects the fixation of the screw. In fact, we have never before found this distribution in cadaver studies or finite element studies. Therefore, the clinical relevance of the present 
study indicates that the placement of the screws in the sacrum is key, in comparison with the fixation in the lumbar vertebra, to obtain a circumferential distribution of the PMMA around the fenestrated screws. In the lumbar spine, although the screw is not placed in the centre of the vertebral body, it is still surrounded by a good layer of cement around it; however, in the sacral vertebra, if the screw is placed too lateral, the cement tends to flow preferentially towards the sacral ala. This is also an indirect indication of the different bone tissue microarchitectures found in lumbar versus sacra vertebra as a possible factor to explain the observed flow differences.

*Limitations of the present study are related to the use of screws with specific fenestration and distribution of holes, as well as with the use of the same acrylic bone cement; distribution of polymeric bone cement may be altered if its specific setting and handling properties are also changed. In this sense, different cements and screws from other manufacturers can generate a different flow distribution and, consequently, the current results and conclusions might not be applied directly to these other fixation systems. Currently, the same type of cemented screw is used in both lumbar and sacral vertebrae. Although in our case the position of the lateral holes was kept constant according to our clinical protocol, the positioning and the number of screw holes can modify the flow and distribution of the cement. In fact this is the factor that must be taken into account to modify this flow in the sacral vertebra. The design of new screws with optimized holes (number, size and position) according to the histomorphometry of the sacral vertebra could achieve a homogeneous flow of cement around these screws.

In the present study, the results indicate that it is necessary to have differentiated and specific injection protocols at least for the sacral vertebra. Evidently, the present results should be confirmed with the analysis of many more cases and experimental clinical studies that can support, in the future, the design of better fixation screws for sacral vertebrae.

However, at present, the main factor that can explain the different flow behaviour of cement has to be related to the different internal bone microarchitectures of both vertebrae. In this sense, neither the vertebrae (different histomorphometric parameters) nor the patients (age, clinical history) are equal to each other. For this reason, all the data obtained is affected by an error. Even within the same vertebra, it is not possible to find identical volumes of interest, with the same histomorphometric parameters. These local specific differences are critical when analysing the flow of cement through porous bone structure. For this reason, our data have an associated error, but this error is directly related to the specific local histomorphometric characteristics of the bone tissue surrounding the screws. 
The distribution of the cement around the screws depends, at first place and for a certain volume of cement injected under the same conditions, on the specific and local histomorphometric characteristics of the receptor porous bone tissue, that is, of the internal microarchitecture of each vertebra that drives the flow of the cement. In this sense, we think that the approach followed in this study can be generalized to different groups of patients with anatomical differences. For example, in the case of deformity, even though the shape of the vertebral body may change and some sclerotic changes may be found at the endplate, this should not greatly affect the trabecular histomorphometry of the vertebral body around the screws. This is, in fact, the key point of the present study, which clearly reveals that the bone cement distribution is less homogeneous around the S1 screws, which should lead to a worse expected fixation. The results obtained indicate that the causes that lead to an inhomogeneous cement flow must be studied. That is why, it is necessary to study the internal microarchitecture of the sacral vertebra. This knowledge should allow to design, in the future, specific infiltration systems for this vertebra.

It should also be noted that if the screw is not placed correctly in the central body of the sacral vertebra, cement is likely to flow into the sacral ala. However, in the present study, as has been commented, the insertion of the pedicle screws was done with the same clinical protocol, with the same angulation, that is, with the same criterion of positioning of the screw relative to the actual size of each vertebra. In no case can it be considered that the final position of the screw can be the critical factor responsible for an inhomogeneous distribution of the cement around the screw.

Finally, in order to find the justification for the observed differences between cement injected in sacral and lumbar vertebrae, a more in deep characterizationincluding a permeability study — of the sacral vertebrae is also required.

\section{Acknowledgments}

The institutional support received through project 2017SGR253 is acknowledged (Agència de Gestiò d'Ajuts Universitaris i de Recerca, Generalitat de Catalunya, Spain).

\section{Funding information}

This work received public funding through project DPI2016-77768-R (Ministerio de Economía y Competitividad, Spain). 
Conflict of interest The authors declare that they have no conflict of interest.

\section{References}

1. Piñera AR, Duran C, Lopez B et al (2011) Instrumented lumbar arthrodesis in elderly patients: prospective study using cannulated cemented pedicle screw instrumentation. Eur Spine J 20(3):408-414. https://doi.org/10.1007/s00586011-1907-2

2. Rajaee SS, Bae HW, Kanim LE, Delamarter RB (2012) Spinal fusion in the United States: analysis of trends from 1998 to 2008. Spine 37(1):67-76. https://doi.org/10.1097/BRS.0b013e31820cccfb

3. Li G, Patil CG, Lad SP et al (2008) Effects of age and comorbidities on complication rates and adverse outcomes after lumbar laminectomy in elderly patients. Spine 33(11):1250-1255. https://doi.org/10.1097/BRS.0b013e3181714a44

4. Raffo CS, Lauerman WC (2006) Predicting morbidity and mortality of lumbar spine arthrodesis in patients in their ninth decade. Spine 31(1):99-103. https://doi.org/10.1097/01.brs.0000192678.25586.e5.

5. Daubs MD, Lenke LG, Cheh G et al (2007) Adult spinal deformity surgery: complications and outcomes in patients over age 60. Spine 32(20):2238-2244. https://doi.org/10.1097/BRS.0b013e31814cf24a

6. Esses SI, Sachs BL, Dreyzin V (1993) Complications associated with the technique of pedicle screw fixation: a selected survey of ABS members. Spine 18(15):2231-2239

7. Polly DW, Orchowski JR, Ellenbogen RG (1998) Revision pedicle screws. Bigger, longer shims - what is best? Spine 23(12):1374-1379

8. von Strempel A, Kühle J, Plitz W (1994) Stability of pedicle screws. 2: maximum pullout force with reference to bone density. Z Orthop Ihre Grenzgeb 132(1):82-86

9. Wang W, Baran GR, Garg H et al (2014) The benefits of cement augmentation of pedicle screw fixation are increased in osteoporotic bone: a finite element analysis. Spine Deform 2(4):248-259.

https://doi.org/10.1016/j.jspd.2014.03.002 
10. Wittenberg RH, Lee KS, Shea M et al (1993) Effect of screw diameter, insertion technique, and bone cement augmentation of pedicular screw fixation strength. Clin Orthop Relat Res 296(296):278-287.

https://doi.org/10.1097/00003086-199311000-00045

11. Pfeifer BA, Krag MH, Johnson C (1994) Repair of failed transpedicle screw fixation: a biomechanical study comparing polymethylmethacrylate, milled bone, and matchstick bone reconstruction. Spine 19(3):350-353

12. Tan JS, Kwon BK, Dvorak MF et al (2004) Pedicle screw motion in the osteoporotic spine after augmentation with laminar hooks, sublaminar wires, or calcium phosphate cement: a comparative analysis. Spine 29(16):1723-1730. https://doi.org/10.1097/01.BRS.0000134569.63542.49

13. Renner SM, Lim TH, Kim WJ et al (2004) Augmentation of pedicle screw fixation strength using an injectable calcium phosphate cement as a function of injection timing and method. Spine 29(11):E212-E216

14. Yi X, Wang Y, Lu H, Li C, Zhu T (2008) Augmentation of pedicle screw fixation strength using an injectable calcium sulphate cement: an in vivo study. Spine 33(23):2503-2509. https://doi.org/10.1097/BRS.0b013e318184e750

15. Liu D, Wu ZX, Pan XM et al (2011) Biomechanical comparison of different techniques in primary spinal surgery in osteoporotic cadaveric lumbar vertebrae: expansive pedicle screw versus polymethylmethacrylate-augmented pedicle screw. Arch Orthop Trauma Surg 131(9):1227-1232.

https://doi.org/10.1007/s00402-011-1290-9.

16. Chang MC, Liu CL, Chen TH (2008) Polymethylmethacrylate augmentation of pedicle screw for osteoporotic spinal surgery: a novel technique. Spine 33(10):E317-E324.

https://doi.org/10.1097/BRS.0b013e31816f6c73

17. Choma TJ, Pfeiffer FM, Swope RW, Hirner JP (2012) Pedicle screw design and cement augmentation in osteoporotic vertebrae: effects of fenestrations and cement viscosity on fixation and extraction. Spine 37(26):E1628-E1632. https://doi.org/10.1097/BRS.0b013e3182740e56

18. Liebschner MAK, Rosenberg WS, Keaveny TM (2001) Effects of bone cement volume and distribution on vertebral stiffness after vertebroplasty. Spine 26(14):1547-1554. https://doi.org/10.1097/00007632-200107150-00009 
19. Elder BD, Lo SF, Holmes C et al (2015) The biomechanics of pedicle screw augmentation with cement. Spine J 15(6):1432-1445.

https://doi.org/10.1016/j.spinee.2015.03.016

20. Blattert TR, Glasmacher S, Riesner HJ, Josten C (2009) Revision characteristics of cement-augmented, cannulated-fenestrated pedicle screws in the osteoporotic vertebral body: a biomechanical in vitro investigation. $\mathrm{J}$ Neurosurg Spine 11(1):23-27. https://doi.org/10.3171/2009.3.SPINE08625

21. Sarzier JS, Evans AJ, Cahill DW (2002) Increased pedicle screw pullout strength with vertebroplasty augmentation in osteoporotic spines. J Neurosurg 96(3):309-312

22. Wilkes RA, Mackinnon JG, Thomas WG (1994) Neurological deterioration after cement injection into a vertebral body. J Bone Joint Surg Br 76(1):155

23. Garg MK, Kharb S (2013) Dual energy X-ray absorptiometry: pitfalls in measurement and interpretation of bone mineral density. Indian J Endocrinol Metab 17(2):203-210. https://doi.org/10.4103/2230-8210.109659

24. Zindrick MR, Wiltse LL, Widell EH et al (1986) A biomechanical study of intrapeduncular screw fixation in the lumbosacral spine. Clin Orthop Relat Res 203:99-112

25. Martín-Fernández M, López-Herradón A, Piñera AR et al (2017) Potential risks of using cement-augmented screws for spinal fusion in patients with low bone quality. Spine J 17(8):1192-1199.

https://doi.org/10.1016/j.spinee.2017.04.029

26. Ebraheim N, Sabry FF, Nadim Y, Xu R, Yeasting RA (2000) Internal architecture of the sacrum in the elderly: an anatomic and radiographic study. Spine 25(3):292-297 